\title{
Cambios en el paisaje ocasionados por incendios forestales en la región de Madera, Chihuahua
}

\section{Wildland fire-induced landscape changes in Madera, Chihuahua region}

\author{
Gabriela Isabel Alva-Álvarez', Humberto Reyes-Hernández², Álvaro Gerardo Palacio-Aponte², \\ Daniel Núñez-López ${ }^{3}$ y Carlos Muñoz-Robles $4^{*}$
}

Universidad Autónoma de San Luis Potosí. Programas Multidisciplinarios de Posgrado en Ciencias Ambientales. San Luis Potosí, SLP, México.

* Autor de correspondencia. carlos.munoz@uaslp.mx

\author{
Universidad Autónoma de San Luis Potosí. Facultad \\ de Ciencias Sociales y Humanidades. San Luis \\ Potosí, SLP, México. \\ ${ }^{3}$ Centro de Investigación en Materiales Avanzados. \\ Unidad Durango. Durango, Dgo., México.
}

\author{
4 Universidad Autónoma de San Luis Potosí. Instituto \\ de Investigación de Zonas Desérticas. San Luis \\ Potosí, SLP, México.
}

\section{RESUMEN}

El fuego es un factor de disturbio que influye en la conformación de los ecosistemas forestales al modificar su estructura. Las alteraciones en el paisaje ocasionadas por incendios forestales pueden ser evaluadas tomando como referencia la condición del paisaje previa al incendio. En este estudio se analizaron los cambios originados por incendios en las características del paisaje en el Municipio de Madera, Chihuahua durante el periodo 2000-2010 y su relación con el área siniestrada. Se delimitaron áreas incendiadas mediante índices espectrales derivados de imágenes Landsdat TM5 y OLI8 y se clasificó la vegetación y uso del suelo dentro de ellos. Se calcularon las métricas del paisaje de área promedio de parches, número de parches, índice del parche más grande y división del paisaje en los polígonos incendiados y se compararon con las métricas del paisaje preincendio. El área promedio de parches y el índice de parche más grande disminuyeron en los paisajes posincendio, mientras que el número de parches y el índice de división del paisaje aumentaron con respecto al paisaje preincendio. El área promedio y el número de parches se correlacionaron con el tamaño de los incendios. Los resultados muestran que después de los incendios los paisajes son más heterogéneos y fragmentados. Las características del paisaje relacionadas con el tamaño de los incendios pueden ser usadas para identificar los paisajes vulnerables a incendios forestales.

PALABRAS CLAVE: BAMS, ecología del paisaje, fragmentación, métricas del paisaje.

\section{ABSTRACT}

Fire is a disturbance factor that influences the formation of forest ecosystems by modifying their structure. Wildland fires alter landscape characteristics that can be evaluated when compared to their pre-fire condition. In this study, wildland fire-induced landscape changes and their relationship with burned extent were analyzed in the region of Madera, Chihuahua during the period 2000-2010. Burned perimeters were delineated using spectral indices derived from Landsat TM5 and OLI8 imagery and the vegetation types within burned areas were classified. Landscape metrics of average patch size, number of patches, largest patch index and landscape division were computed within burned polygons and compared with pre-fire landscape metrics. Average patch size and the largest patch index decreased after wildland fires, whilst number of patches and landscape division increased in comparison with pre-fire conditions. Average patch size and the number of patches were correlated with burned area. The results show that landscapes are more heterogeneous and fragmented after the occurrence of wildland fires. Landscape characteristics related to burned area can be used to identify fire-prone landscapes.

KEYWORDS: BAMS, landscape ecology, fragmentation, landscape metrics. 


\section{INTRODUCCIÓN}

El fuego es un elemento natural que influye en el mantenimiento, función y evolución de los ecosistemas, además de ser un componente importante en las interacciones entre los patrones y procesos que se desarrollan a nivel de paisaje (Parisien, Miller, Ager y Finney, 2010). Los incendios forestales son un factor de disturbio que impacta de distintas maneras a los ecosistemas forestales en función de su frecuencia y su severidad (Di Bella et al., 2008), entendida esta última como el grado de cambio en el suelo y la pérdida de vegetación y materia orgánica causados por el fuego (Keeley, 2009). Por otra parte, los incendios forestales tienen una dimensión espacial que se caracteriza por su ocurrencia en el territorio (Lloret, Calvo, Pons y Díaz-Delgado, 2002), y su incidencia es uno de los principales factores que explica el desarrollo de paisajes altamente combustibles, como consecuencia de la continuidad y uniformidad espacial de la propia estructura del paisaje (Pérez-Cabello et al., 2007).

Analizar los impactos del fuego sobre el paisaje permite comprender e interpretar el origen de los patrones del paisaje actual y facilita responder adecuadamente con medidas correctoras a los efectos negativos en las zonas incendiadas (Bajocco y Ricotta, 2008). Existe evidencia de que el fuego tiene efectos en los patrones del paisaje y viceversa (Haire y McGarical, 2010). Al respecto, se han desarrollado investigaciones que abordan los cambios en el paisaje como consecuencia de los incendios forestales, y se ha encontrado que los incendios forestales repercuten directamente en los patrones de distribución de la vegetación y la fragmentación del paisaje (Bizama et al., 2011). En algunos casos, la diversidad de paisaje presenta una mayor homogeneidad tras los incendios (Pérez-Cabello et al., 2007; Vázquez de la Cueva y Rodríguez Martín, 2008), pero también, la diversidad del paisaje a largo plazo puede aumentar como consecuencia del fuego (Silva, Vaz, Moreira, Catry y Rego, 2011).

Un aspecto de gran relevancia es el análisis de las relaciones entre la magnitud de los incendios y los patrones del paisaje, aunque ha sido poco estudiado. En este sentido, se ha encontrado que la heterogeneidad del paisaje está en función del tamaño de los incendios y que a su vez, el mosaico que forma el paisaje, determina de manera importante el tamaño de los incendios (Haire y McGarical, 2010; Ireland, Stan y Fulé, 2012). El conocimiento de estas interacciones es útil para clasificar paisajes vulnerables a incendios, que como resultado de su configuración espacial preincendio propician su ocurrencia y la consecuente propagación del fuego (Lavorel, Flanningan, Lambin y Scholes, 2007).

No obstante que el fuego es un componente natural en diversos ecosistemas terrestres, los incendios en México son un factor de perturbación que causa daños en los ecosistemas forestales y en poco tiempo pueden afectar a grandes áreas (Flores y Benavides, 1994). Chihuahua es uno de los estados con mayor ocurrencia de incendios con base en datos de la Comisión Nacional Forestal [Conafor] (2016); en el periodo 1998-2016, se presentó un promedio de 900 incendios por año, lo que equivale a 11\% del total de incendios registrados anualmente en el país. Estos incendios impactaron alrededor de 21600 ha anuales de bosques con renuevo y arbolado adulto, matorrales y pastizales $\mathrm{y}$, en función de su severidad, han alterado el paisaje en distinta magnitud.

En México, el conocimiento sobre la manera en que los patrones del paisaje predisponen la ocurrencia de incendios forestales y cómo estos, a su vez, modifican el paisaje es escaso. El estudio de las interacciones fuegopaisaje da una pauta indispensable que permite conocer las características de los paisajes más vulnerables al fuego y complementa los planes de manejo, conservación y restauración forestal.

\section{OBJETIVOS}

El objetivo de este trabajo fue analizar las interacciones entre los incendios forestales y el paisaje en el Municipio de Madera en el periodo 2000-2010. Para alcanzar este objetivo, fue necesario: 1) cuantificar la magnitud de los cambios en los patrones del paisaje (área promedio de parches, número de parches, índice del parche más grande, índice de división del paisaje) ocasionados por incendios forestales y 2) identificar las características del paisaje que 
se relacionan con el tamaño de los polígonos incendiados. Como hipótesis, se espera que haya mayor fragmentación y homogeneidad y menor conectividad dentro de los polígonos incendiados. Asimismo, se espera que las métricas del paisaje sean variables predictoras del tamaño de los polígonos incendiados, ya que la propagación del fuego depende en gran medida del arreglo espacial de los combustibles forestales y existe una relación estrecha entre estos y el tipo de vegetación (Burgan, Klaver y Klaver, 1998; Turner y Romme, 1994).

\section{MATERIALES Y MÉTODOS}

\section{Descripción del área de estudio}

El municipio de Madera, Chihuahua, se localiza en la Sierra Madre Occidental, entre las coordenadas $30^{\circ} 40^{\prime} 0$ " y $25^{\circ}$ $30^{\prime} 56^{\prime \prime}$ de latitud norte y entre $109^{\circ} 6^{\prime} 30^{\prime \prime}$ y $104^{\circ} 54^{\prime} 08^{\prime \prime}$ de longitud oeste y se extiende sobre 815200 ha (Fig. 1). El área de estudio se encuentra en la provincia fisiográfica Sierra Madre Occidental, dentro de la subprovincia Sierras y Cañadas del Norte, con una elevación promedio de 2500 $\mathrm{m}$, con puntos en donde alcanza $3300 \mathrm{~m}$ snm. Predominan sierras de laderas escarpadas constituidas en su mayoría por rocas volcánicas ácidas, entre las cuales se localizan valles inter-montanos (Instituto Nacional de Estadística y Geografía [Inegi], 2003). En el área de estudio se presentan climas semifríos subhúmedos $\mathrm{Cb}^{\prime}(\mathrm{w} 2) \mathrm{x}^{\prime}$, templados subhúmedos $\mathrm{C}($ w1) x' y $\mathrm{C}$ (wo)x' y semi-áridos semicálidos BS1h( $\left.\mathrm{x}^{\prime}\right)$, de acuerdo con la clasificación de Köppen modificada por García (1973). La temperatura media anual es de $17^{\circ} \mathrm{C}$, mientras que la precipitación, que se presenta en los meses de verano, oscila entre $800 \mathrm{~mm}$ y $1500 \mathrm{~mm}$. Los principales tipos de vegetación que se encuentran en el área de estudio son bosque de encino, bosque de pinoencino, bosque de pino, bosque de encino-pino y bosques de oyamel (Palacio et al., 2000). Las principales especies en estas comunidades son Pinus engelmannii, $P$. durangensis, $P$. arizonica, $P$. leiophylla, $P$. ayacahuite, $P$. herreraii, Abies religiosa y Pseudotsuga menziessi, Quercus sideroxyla, Q. arizonica y Q. rugosa. En menor proporción se encuentran chaparral, pastizal, selva baja caducifolia y áreas agrícolas. La producción maderable es una de las principales actividades económicas, los bosques se aprovechan bajo esquemas silvícolas de bosques regulares para bosques de pino y de bosques irregulares para bosques de pino-encino y bosques de encino.

\section{Metodología}

\section{Elementos del paisaje}

Para el presente trabajo, el paisaje se consideró como un área heterogénea cuyo patrón espacial es recurrente en el territorio y está compuesto por ecosistemas, tipos de vegetación y uso del suelo que interactúan entre si (Forman y Godron, 1986; Green, Simmons y Woltjer, 1996). De esta manera, los tipos de vegetación se consideraron como los elementos del paisaje y se analizaron los cambios ocurridos en sus atributos espaciales dentro cada polígono incendiado; estos polígonos, a su vez, constituyeron paisajes independientes para su análisis. El estudio consistió de cinco etapas: 1) obtención y pre-procesamiento de imágenes de satélite, 2) delimitación de polígonos incendiados, 3) clasificación de la vegetación preincendio y posincendio en los polígonos incendiados, 4) cálculo de métricas del paisaje y 5) comparación de paisajes preincendio y posincendio.

\section{Descripción de imágenes de satélite}

Se obtuvieron del portal GloVis (United States Department of the Interior, s/f) 46 imágenes de los satélites Landsat TM 5 y Landsat OLI 8 (Path 33 y 34, Row 39 y 40) del periodo 1999-2010 y una del año 2014 de los meses de enero a marzo, periodo que corresponde aproximadamente al inicio de la temporada de incendios. Esto permitió identificar áreas antes y después de los incendios, como se describe en la siguiente sección. Las imágenes tuvieron una resolución espacial de $30 \mathrm{~m}$ y fueron referidas a la proyección Cónica Conforme de Lambert en virtud de que el área de estudio está comprendida en dos zonas UTM, lo que imposibilitó su correcta unión geográfica. La geo-referencia se realizó con un error de posición < $30 \mathrm{~m}$ para asegurar una adecuada sobreposición espacial de las imágenes. 


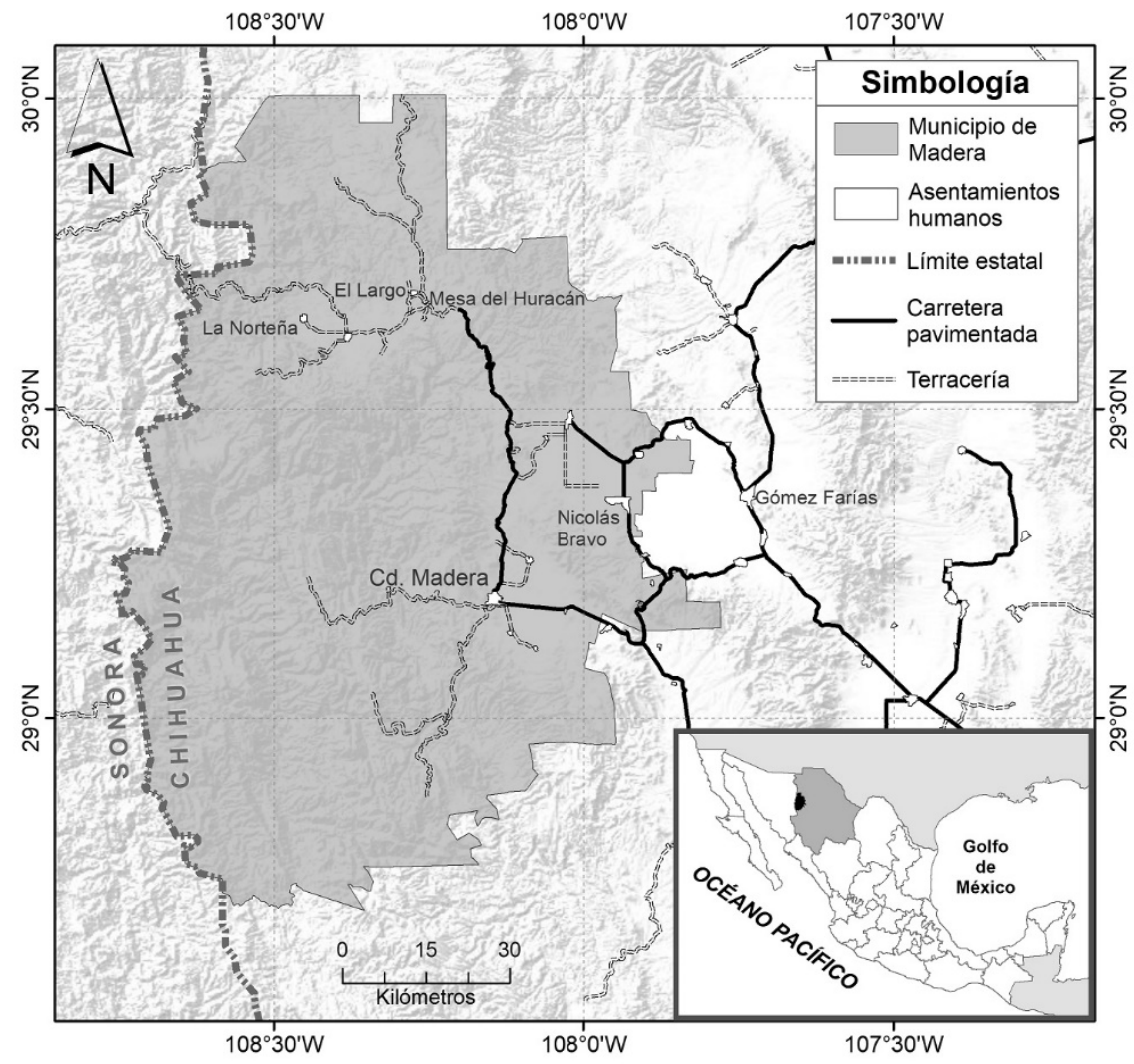

FIGURA 1. Localización del área de estudio.

\section{Delimitación polígonos quemados}

Se utilizó el programa BAMS (Burned Area Mapping) para identificar las áreas quemadas (Bastarrika et al., 2014). El primer paso consistió en el procesamiento individual de cada escena Landsat con nivel 1T (i. e. números digitales que pueden ser calibrados a valores de reflectancia usando los metadatos incluidos en cada escena) en formato GeoTiff para estimar la reflectancia exo-atmosférica (TOA por sus siglas en inglés) y minimizar el efecto de la atmósfera entre las distintas fechas de toma de las imágenes. Las áreas incendiadas se caracterizan por generar depósitos de carbón y cenizas, así como por la remoción de la vegetación y la exposición de la superficie del suelo. Estos factores dependen del tipo y condición de la vegetación previa al incendio y del nivel de combustión alcanzado después del incendio (Roy y Landmann, 2005) y pueden identificarse mediante el uso de índices espectrales (Masek et al., 2013). Para esto, se tuvieron pares de escenas correspondientes a la condición previa al incendio y posterior al incendio para cada año. Por ejemplo, la imagen de 1999 fungió como imagen preincendio y la imagen 2000 como imagen posincendio; la imagen de 2000 como imagen preincendio y la imagen de 2001 como imagen posincendio, y así sucesivamente hasta el 2010. En un segundo paso, BAMS calculó los índices espectrales relevantes para identificar estos cambios (Tabla 1).

Estos índices se calcularon para las imágenes preincendio y posincendio, además se calculó el diferencial de cada uno de ellos, con lo que se generaron series temporales que permitieron discriminar las áreas incendiadas con base en las diferencias espectrales de las condiciones preincendio y posincendio. 
TABLA 1. Índices espectrales usados por el programa BAMS para discriminar áreas incendiadas.

\begin{tabular}{lc}
\hline Índice espectral & Ecuación \\
\hline Normalized Difference Vegetaton Index & $N D V I=\frac{\rho_{\text {NIR }}-\rho_{R E D}}{\rho_{\text {NIR }}+\rho_{R E D}}$ \\
Modified Burned Area Mapping Index & $B A I M=\frac{1}{\left(\rho_{N I R}-0.05\right)^{2}+\left(\rho_{\text {SWIRI }}-0.2\right)^{2}}$ \\
Global Environmental Monitoring Index & $G E M I=\eta(1-0.25 \eta)-\frac{\rho_{R E D-0.125}}{1-\rho_{R E D}}$ \\
Normalized Burned Ratio & $N B R=\frac{\rho_{\text {NIR }}-\rho_{\text {SWIRL }}}{\rho_{\text {NIR }}+\rho_{\text {SWIRL }}}$ \\
Burned Index & $M I R B I=10 \rho_{\text {SWIRL }}-9.8 \rho_{\text {SWIRS }}$ \\
\hline
\end{tabular}

Donde: $\eta=\left(2\left(\rho_{N I R^{2}}-\rho_{R E D^{2}}\right)+1.5 \rho_{N I R}+0.5 \rho_{R E D}\right) /\left(\rho_{N I R}+\rho_{R E D}+0.5\right) ; \rho_{R E D}=$ Reflectancia en la banda roja; $\rho_{N I R}=$ Reflectancia en el infrarojo cercano 1 ; $\rho$ swIRS $=$ Reflectancia en el infrarojo cercano 2 (con centro de longitud de onda de alrededor de $1.6 \mu \mathrm{m}$ ); $\rho_{\text {swIRL }}=$ Reflectancia en el infrarojo medio (con centro de longitud de onda de alrededor de $2.2 \mu \mathrm{m}$ )
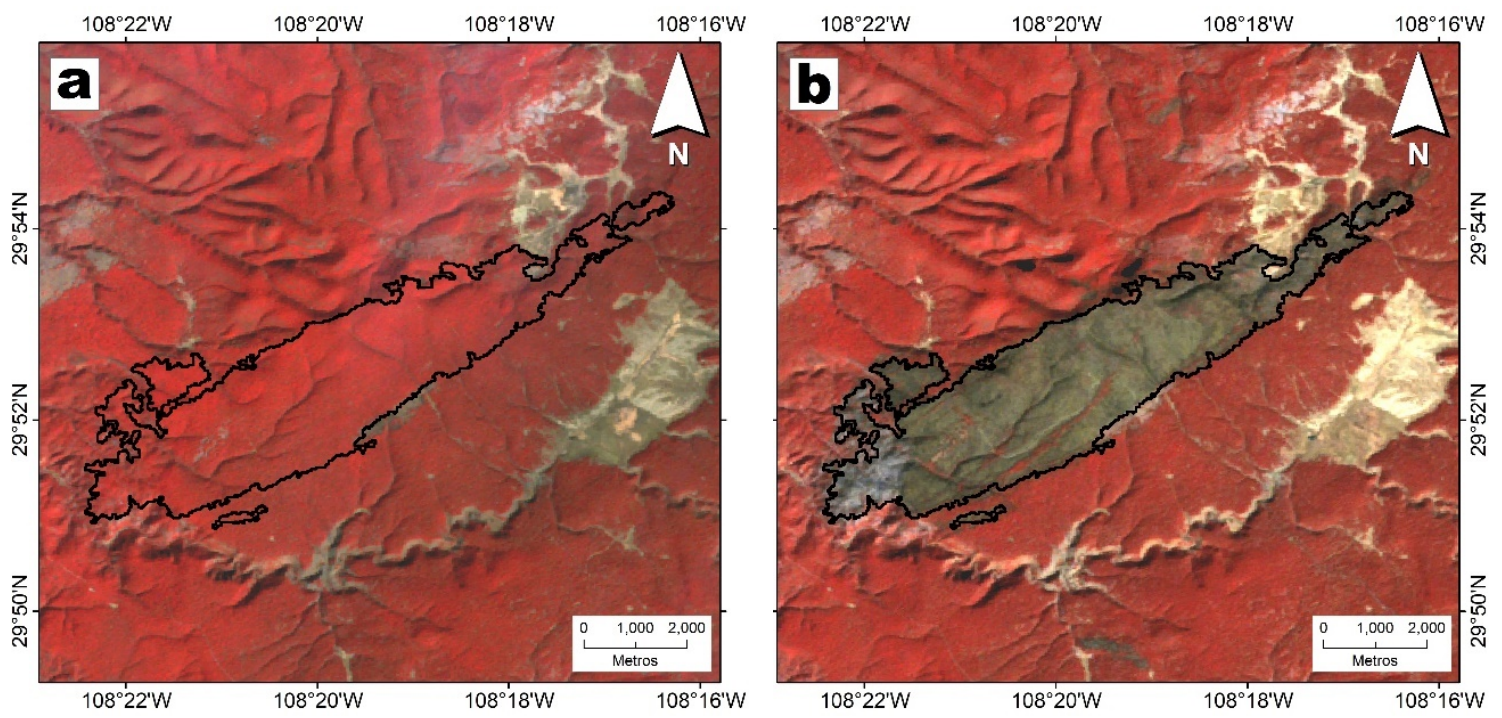

FIGURA 2. Ejemplo del resultado de la delimitación de un área incendiada con el programa BAMS.

(a) Imagen preincendio y (b) imagen posincendio. Las imágenes son compuestos en falso color con la combinación de bandas 4-3-1 (oNIR-RED-BLUE).

El último paso en BAMS consistió en la delimitación de áreas quemadas. A partir de las imágenes preincendio y posincendio, se crearon áreas de entrenamiento de manera visual en las imágenes para caracterizar las áreas quemadas con base en cinco índices espectrales (Tabla 1). Posteriormente, el programa realizó un análisis en dos fases; la primera consistió en identificar y mantener los pixeles de la imagen que claramente se muestran como áreas quemadas. En la segunda, se analizaron los pixeles vecinos que tenían respuestas espectrales similares a los pixeles identificados como quemados y se incluyeron progresivamente hasta generar los polígonos de las áreas incendiadas en formato vectorial (Fig. 2).

\section{Clasificación de imágenes de satélite}

Con el fin de conocer las características del paisaje e identificar sus cambios como consecuencia de incendios, el periodo de estudio se dividió en lapsos de alrededor de cinco años de amplitud. Los tipos de vegetación y uso del suelo de las imágenes de los años 2005, 2009 y 2014 se 
utilizaron como intervalos de tiempo de referencia para el análisis de los cambios en el paisaje. Para esto, los incendios ocurridos de 2000 a 2003 se evaluaron con la clasificación de la imagen de 2005, los incendios ocurridos entre 2004 y 2007 con la clasificación de la imagen 2009 y los incendios ocurridos de 2008 a 2010 con la clasificación de 2014. La elección de estos periodos de tiempo permitió identificar las áreas quemadas en cada año y comparar las características del paisaje preincendio con imágenes que abarcaron entre uno y cinco años después de los incendios, ya que en este periodo de tiempo, los efectos del fuego en la vegetación y el suelo aún persisten en el paisaje (Robichaud, Beyers y Neary, 2000).

Se realizaron clasificaciones supervisadas empleando el algoritmo de máxima verosimilitud (Maximum likelihood) en el programa ENVI 5.3. Este algoritmo analiza y asigna cada pixel a la clase con la que muestra una mayor probabilidad de pertenencia (Chuvieco, 1996). Se utilizaron 724 puntos de referencia para cada tipo de vegetación y uso del suelo, tomados a partir de Google Earth. Se definieron tres tipos de vegetación: bosque de pino, bosque de encino, bosque de pino-encino; y un tipo de uso del suelo: agricultura. Además, se utilizaron 323 puntos de verificación tomados de las series III, IV y V de vegetación y uso del suelo de Inegi para validar las clasificaciones del 2005, 2009 y 2014, respectivamente. Se elaboraron matrices de error para estimar la fiabilidad de las clasificaciones y determinar el porcentaje de pixeles clasificados correctamente (Arenas, Haeger y Jordano, 2011). También, se calculó el estadístico de Kappa, que es una medida de concordancia que mide la diferencia entre la exactitud lograda en la clasificación con un clasificador automático y la probabilidad de lograr una clasificación correcta con un clasificador aleatorio. Los valores del coeficiente de Kappa van de 0 a 1; entre más cercano a 1 es el valor de este coeficiente, mayor es la exactitud del mapa con respecto a la realidad (Chuvieco, 1996).

\section{Métricas del paisaje}

Para cada polígono incendiado (2000-2010), se extrajo el tipo de vegetación de sus condiciones preincendio y posincendio y se calcularon métricas del paisaje del bosque de pino, bosque de pino-encino y bosque de encino usando la extensión LecoS del programa Quantum Gis (Jung, 2016). Las categorías de áreas agrícolas y cuerpos de agua no fueron consideradas para el análisis, ya que constituían $<0.1 \%$ del total del área de los polígonos incendiados. Las métricas calculadas dentro de cada polígono incendiado fueron el área promedio de parches, número de parches, índice del parche más grande e índice de división del paisaje (Tabla 2). Los análisis fueron elaborados para cada periodo descrito en la sección anterior (1año - 5 años después del incendio).

TABLA 2. Métricas del paisaje calculadas a nivel de clase (i. e. tipo de vegetación) dentro de los polígonos incendiados.

\begin{tabular}{ll}
\hline Métrica & Descripción \\
\hline Área promedio de parches & Representa el área promedio por cada tipo de parche. \\
Número de parches & Indica el número de parches en cada polígono. \\
Índice del parche más grande & $\begin{array}{l}\text { Cuantifica el porcentaje de área total ocupado por el parche } \\
\text { más grande. }\end{array}$ \\
& $\begin{array}{l}\text { Mide la probabilidad de que dos pixeles elegidos al azar no } \\
\text { Índice de división del paisaje }\end{array}$ \\
\end{tabular}




\section{Análisis de datos}

Con la finalidad de tener una medida de correspondencia entre las superficies de los polígonos identificados con BAMS y las áreas de los incendios ocurridos en el terreno, se realizó un análisis de correlación entre las superficies de las áreas quemadas en cada año dentro del periodo de estudio y las áreas de los incendios registrados por la Comisión Nacional Forestal (Conafor, 2016). Los cambios en el paisaje dentro de los polígonos incendiados se evaluaron con la prueba no-paramétrica de Wilcoxon pareada debido a que los datos de las métricas del paisaje no seguían una distribución normal incluso con datos transformados. Con esta prueba se compararon las métricas de los polígonos con condición preincendio y posincendio a nivel de tipo de vegetación para los tres lapsos de tiempo considerados. Para identificar las métricas del paisaje preincendio que están relacionadas con el área de los polígonos incendiados se realizó un análisis de correlación de Spearman, seguido de esto se generó un modelo de regresión lineal para evaluar la proporción de la varianza del área de los incendios que es explicada por las métricas del paisaje. Este modelo incluyó únicamente las métricas correlacionadas con el área de los polígonos incendiados luego de verificar que no estuvieran correlacionadas entre sí para evitar redundancia en el modelo de regresión. Los valores de significancia fueron establecidos como $\mathrm{P}<0.05$ para todos los análisis estadísticos, mismos que se realizaron en el programa R (R Development Core Team, 2015).

\section{Resultados}

\section{Áreas incendiadas y clasificación de la vegetación}

Se identificaron 384 polígonos incendiados, que sumaron un área de 8277 ha. En promedio, se detectaron 35 incendios por año con tasa anual de quema de $9 \%$. El tamaño de los incendios varió entre 3.24 ha y 1692 ha $(\bar{x}=$ $20.60 \mathrm{ha})$, los incendios más frecuentes tuvieron un tamaño entre 4 ha y 40 ha. Los incendios ocurrieron en su mayoría en la parte norte del municipio de Madera. La correlación entre las áreas incendiadas identificadas en este trabajo y las áreas obtenidas por Conafor fue significativa y alta $(\mathrm{R}=$ 0.89; $\mathrm{P}<0.0002)$, lo que es consistente en términos del área real quemada y el área obtenida de los polígonos incendiados delimitados en este trabajo.

La fiabilidad global de las clasificaciones osciló entre $88 \%$ y $92 \%$ (Tablas 3, 4 y 5), lo que indicó una adecuada identificación de los tipos de vegetación, mientras que el estadístico de Kappa alcanzó valores entre 0.86 y 0.90 y mostró que las clasificaciones fueron al menos $86 \%$ mejores que la confiabilidad esperada si se hubiera asignando de manera aleatoria una categoría de cobertura a los polígonos clasificados.

TABLA 3. Matriz de confusión para la clasificación de la vegetación y uso del suelo de 2005 (Fiabilidad global = 90\%; Estadístico de Kappa = 0.87).

\begin{tabular}{|c|c|c|c|c|c|c|c|c|}
\hline Vegetación/uso del suelo & $\begin{array}{c}\text { Bosque } \\
\text { de } \\
\text { encino }\end{array}$ & $\begin{array}{l}\text { Bosque } \\
\text { de pino }\end{array}$ & $\begin{array}{l}\text { Bosque de } \\
\text { pino-encino }\end{array}$ & Agrícola & $\begin{array}{c}\text { Cuerpo de } \\
\text { agua }\end{array}$ & $\begin{array}{c}\text { Sin } \\
\text { vege- } \\
\text { tación }\end{array}$ & $\begin{array}{c}\text { Fiabilidad } \\
\text { del } \\
\text { usuario } \\
(\%)\end{array}$ & $\begin{array}{c}\text { Error de } \\
\text { comisión } \\
\text { (\%) }\end{array}$ \\
\hline Bosque de encino & 16 & 1 & 2 & 0 & 0 & 1 & 80.0 & 20.0 \\
\hline Bosque de pino & 2 & 15 & 1 & 0 & $\mathrm{O}$ & $\mathrm{O}$ & 83.3 & 16.7 \\
\hline Bosque de pino-encino & 2 & 2 & 18 & 0 & 0 & 0 & 81.8 & 18.2 \\
\hline Agrícola & 0 & 0 & 0 & 17 & 0 & 0 & 100.0 & 0.0 \\
\hline Cuerpo de agua & 0 & 0 & $\mathrm{O}$ & 0 & 17 & 0 & 100.0 & 0.0 \\
\hline Sin vegetación & 0 & 0 & $\mathrm{O}$ & O & 0 & 15 & 100.0 & 0.0 \\
\hline Fiabilidad del productor (\%) & 80.0 & 83.3 & 85.7 & 100.0 & 100.0 & 93.8 & & \\
\hline Error de omisión (\%) & 20.0 & 16.7 & 14.3 & 0.0 & 0.0 & 6.3 & & \\
\hline
\end{tabular}


TABLA 4. Matriz de confusión para la clasificación de la vegetación y uso del suelo de 2009 (Fiabilidad global = 92\%; Estadístico de Kappa $=0.90)$.

\begin{tabular}{|c|c|c|c|c|c|c|c|c|}
\hline Vegetación/uso del suelo & $\begin{array}{c}\text { Bosque } \\
\text { de } \\
\text { encino }\end{array}$ & $\begin{array}{l}\text { Bosque } \\
\text { de pino }\end{array}$ & $\begin{array}{c}\text { Bosque de } \\
\text { pino-encino }\end{array}$ & Agrícola & $\begin{array}{c}\text { Cuerpo de } \\
\text { agua }\end{array}$ & $\begin{array}{c}\text { Sin } \\
\text { vege- } \\
\text { tación }\end{array}$ & $\begin{array}{c}\text { Fiabilidad } \\
\text { del } \\
\text { usuario } \\
\text { (\%) } \\
\end{array}$ & $\begin{array}{c}\text { Error de } \\
\text { comisión } \\
\text { (\%) }\end{array}$ \\
\hline Bosque de encino & 20 & 0 & 2 & 0 & 0 & 2 & 83.3 & 16.7 \\
\hline Bosque de pino & 2 & 18 & 0 & 0 & 0 & 0 & 90.0 & 10.0 \\
\hline Bosque de pino-encino & 0 & 3 & 16 & 0 & 0 & 0 & 84.2 & 15.8 \\
\hline Agrícola & 0 & 0 & 0 & 17 & 0 & 0 & 100.0 & 0.0 \\
\hline Cuerpo de agua & 0 & 0 & 0 & 0 & 17 & 0 & 100.0 & 0.0 \\
\hline Sin vegetación & 0 & 0 & 0 & 0 & 0 & 15 & 100.0 & 0.0 \\
\hline Fiabilidad del productor (\%) & 90.9 & 85.7 & 88.9 & 100.0 & 100.0 & 88.2 & & \\
\hline Error de omisión (\%) & 9.1 & 14.3 & 11.1 & 0.0 & 0.0 & 11.8 & & \\
\hline
\end{tabular}

TABLA 5. Matriz de confusión para la clasificación de la vegetación y uso del suelo de 2014 (Fiabilidad global = 88\%; Estadístico de Kappa = 0.86).

\begin{tabular}{|c|c|c|c|c|c|c|c|c|}
\hline Vegetación/uso del suelo & $\begin{array}{c}\text { Bosque } \\
\text { de } \\
\text { encino }\end{array}$ & $\begin{array}{l}\text { Bosque } \\
\text { de pino }\end{array}$ & $\begin{array}{l}\text { Bosque de } \\
\text { pino-encino }\end{array}$ & Agrícola & $\begin{array}{c}\text { Cuerpo de } \\
\text { agua }\end{array}$ & $\begin{array}{c}\text { Sin } \\
\text { vege- } \\
\text { tación }\end{array}$ & $\begin{array}{c}\text { Fiabilidad } \\
\text { del } \\
\text { usuario } \\
\text { (\%) }\end{array}$ & $\begin{array}{c}\text { Error de } \\
\text { comisión } \\
\text { (\%) }\end{array}$ \\
\hline Bosque de encino & 15 & 1 & 1 & 0 & 0 & 2 & 88.2 & 11.8 \\
\hline Bosque de pino & 0 & 14 & 3 & 0 & 0 & 0 & 82.4 & 17.6 \\
\hline Bosque de pino-encino & 2 & 2 & 13 & 0 & 0 & 1 & 76.5 & 23.5 \\
\hline Agrícola & 0 & 0 & $\mathrm{O}$ & 17 & 0 & $\mathrm{O}$ & 100.0 & 0.0 \\
\hline Cuerpo de agua & 0 & 0 & 0 & 0 & 17 & 0 & 100.0 & 0.0 \\
\hline Sin vegetación & $\mathrm{O}$ & 0 & 0 & 0 & 0 & 14 & 100.0 & 17.6 \\
\hline Fiabilidad del productor (\%) & 78.9 & 82.4 & 72.2 & 100.0 & 100.0 & 100.0 & 88.9 & \\
\hline Error de omisión (\%) & 21.1 & 17.6 & 27.8 & 0.0 & 0.0 & 0.0 & 11.1 & \\
\hline
\end{tabular}

El tipo de vegetación que predominó antes de los incendios en los polígonos quemados fue el bosque de pino-encino, seguido en orden ascendente por el bosque de pino y el bosque de encino (Fig. 3).

\section{Características del paisaje preincendio contra paisaje posincendio}

Las características del paisaje preincendio y posincendio fueron diferentes en la mayoría de los casos y la tendencia general indicó el aumento del número de parches y la disminución de su área dentro de los polígonos incendiados (Fig. 4). El área promedio de los parches disminuyó después de los incendios ( $\mathrm{P}<0.0001$; Fig. $5 \mathrm{~A}$ ). El área promedio de los parches de bosque de pino y bosque de pino-encino se redujo por poco más de un orden de magnitud, mientras que el área promedio de los parches de bosque de encino disminuyó 2.3 veces con respecto a la condición preincendio. Por otra parte, el número de parches aumentó después de los incendios ( $\mathrm{P}<0.0001$; Fig. 5B). La cantidad de parches de bosque de pino y bosque de pino-encino 
aumentaron alrededor de tres veces con respecto a la condición preincendio, mientras que los parches de bosque de encino aumentaron cuatro veces su número después de los incendios.

El índice del parche más grande fue mayor en el periodo preincendio comparado con el periodo posincendio para los parches de bosque de pino y bosque de pino-encino $(\mathrm{P}<0.0001$; Fig. $5 \mathrm{C})$, lo que reflejó un número de parches entre tres y cuatro veces más pequeños después de los incendios, excepto para los parches del bosque de encino, donde no se encontró diferencia del índice del parche más grande entre los paisajes preincendio y posincendio $(\mathrm{P}=0.208)$.

La división del paisaje incrementó en los paisajes posincendio $(\mathrm{P}<0.0001$; Fig. 5D), en mayor magnitud el bosque de pino y en el bosque de pino-encino, lo que reveló mayor discontinuidad espacial entre los tres tipos de vegetación luego de la ocurrencia de incendios.

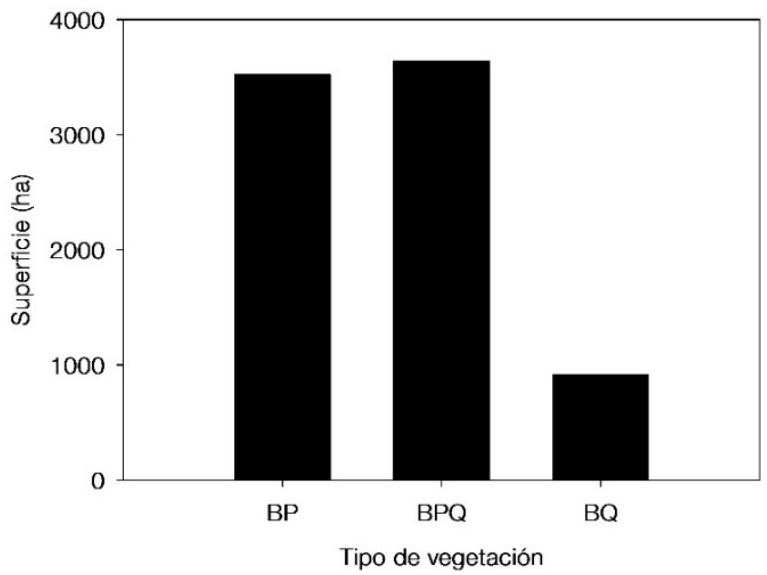

FIGURA 3. Tipo de vegetación preincendio en los polígonos incendiados en el periodo 2000-2010.

$\mathrm{BP}=$ Bosque de pino, $\mathrm{BPQ}=$ Bosque de pino-encino, $\mathrm{BQ}=$ = Bosque de encino.
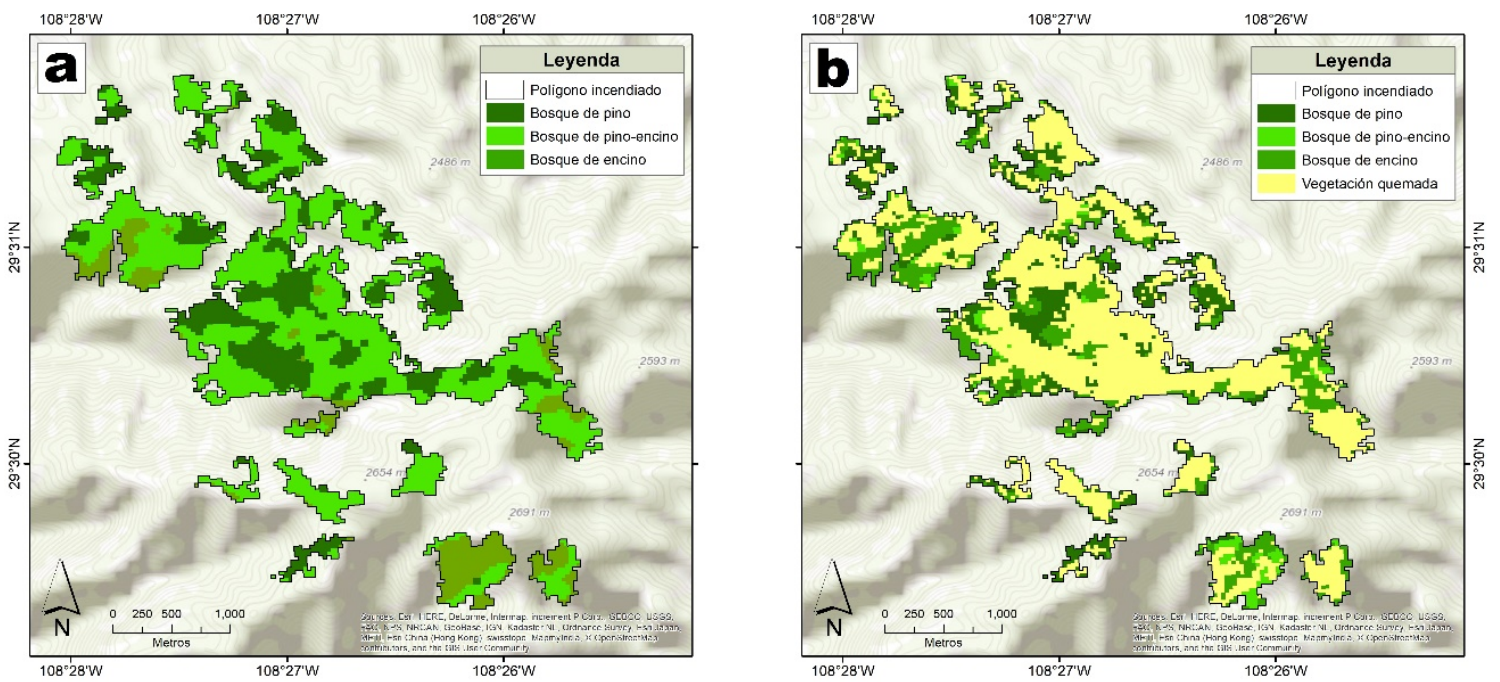

FIGURA 4. Ejemplo de la configuración del paisaje para incendios ocurridos en el periodo 2000-2003.

(a) paisaje preincendio y (b) paisaje posincendio.

Puede observarse la disminución del área de parches y el aumento del número de parches en el paisaje posincendio en contraste con el paisaje preincendio. 

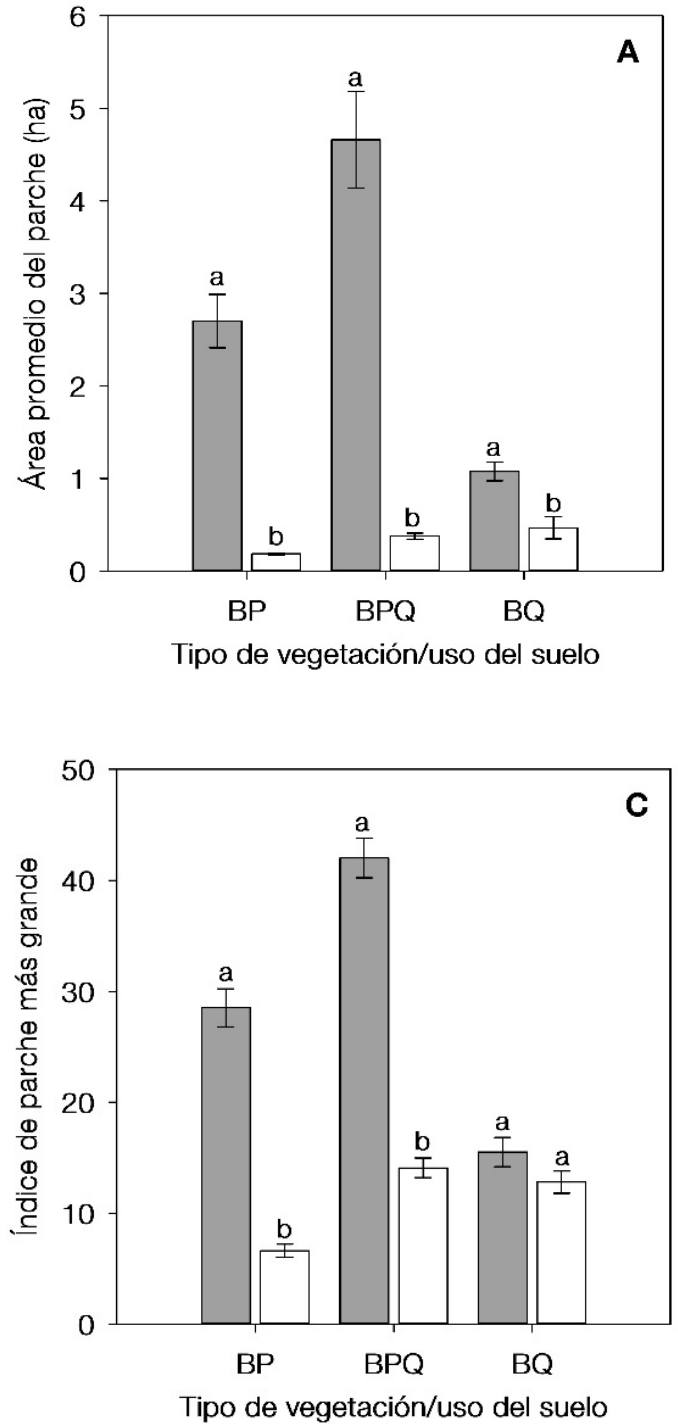
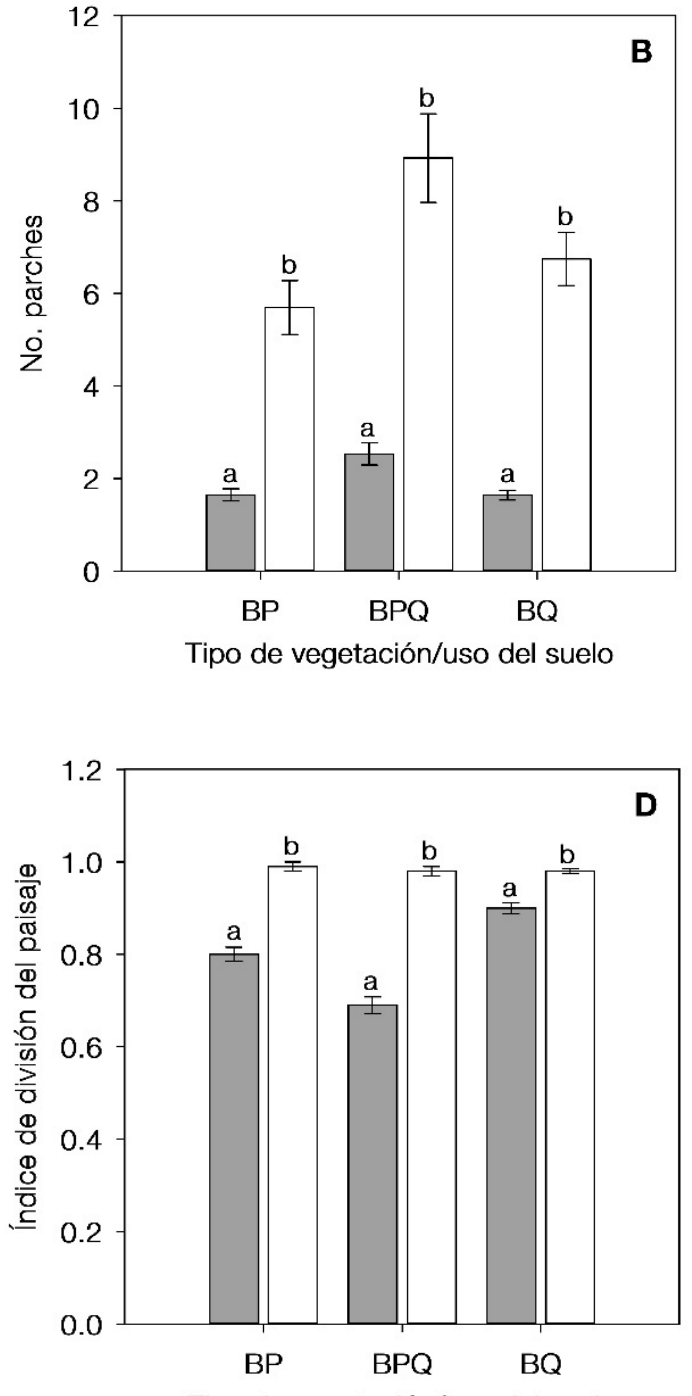

Tipo de vegetación/uso del suelo

FIGURA 5. Características promedio del paisaje preincendio (barras grises) y posincendio (barras en blanco).

A) Área promedio del parche; B) número de parches; C) índice del parche más grande; D) Índice de división del paisaje. Letras diferentes indican diferencias significativas $(P<0.05)$ entre cada métrica dentro de cada tipo de vegetación analizado. $\mathrm{BP}=$ Bosque de pino, $\mathrm{BPQ}=$ Bosque de pino-encino, $\mathrm{BQ}=$ Bosque de encino.

\section{Relaciones entre el área incendiada y las caracte- rísticas del paisaje preincendio}

Las métricas del paisaje preincendio más correlacionadas con el área incendiada fueron el número de parches de los tres tipos de vegetación y el área promedio de parches de bosque de pino-encino (Tabla 6). Todas las correlaciones significativas fueron positivas, es decir, a mayor número y área de parches de estos tipos de vegetación, el área del polígono incendiado aumentó.

En la base de datos de los polígonos incendiados delimitados con el programa BAMS se detectó un incendio con una extensión poco común de 1692 ha. Las bases de datos de incendios de la Conafor confirman un incendio de este tamaño. Aunque dicho incendio ocurrió, constituye un 
valor atípico, ya que las áreas de incendios registrados oscilan entre 1 ha y 300 ha. Por esta razón, este registro fue eliminado antes de generar el modelo de regresión lineal. El modelo para predecir el área incendiada, resultante de la integración de las métricas correlacionadas con el tamaño del polígono incendiado explicó $78 \%$ de la variabilidad en el área de los polígonos incendiados con área menor a 300 ha (Fig. 6) y se expresó como:

\section{Área incendiada}

$$
\begin{aligned}
& =-7.91+\left(4.68 \times N P_{-} B P\right) \\
& +\left(0.76 \times A P_{-} B P Q\right)+\left(3.79 \times N P_{-} B P Q\right) \\
& +\left(2.62 \times N P_{-} B Q\right)
\end{aligned}
$$

donde:

NP_BP $=$ Número de parches de bosque de pino

AP_BPQ =Área promedio de parches de bosque de pinoencino

NP_BPQ =Número de parches de bosque de pino-encino

NP_BQ = Número de parches de bosque de encino

TABLA 6. Coeficientes de correlación de Spearman entre el área incendiada y las métricas del paisaje calculadas a nivel de clase (i. e. tipo de vegetación).

\begin{tabular}{lc}
\hline Área promedio de parches-Bosque de pino & 0.2 \\
\hline Número de parches-Bosque de pino & 0.77 \\
\hline Índice del parche más grande-Bosque de pino & 0 \\
\hline División del paisaje-Bosque de pino & 0 \\
\hline Área promedio de parches-Bosque de pino-encino & 0.42 \\
\hline Número de parches-Bosque de pino-encino & 0.63 \\
\hline Índice del parche más grande-Bosque de pino-encino & 0.07 \\
\hline División del paisaje-Bosque de pino-encino & -0.07 \\
\hline Área promedio de parches-Bosque de encino & 0.13 \\
\hline Número de parches-Bosque de encino & 0.56 \\
\hline Índice del parche más grande-Bosque de encino & -0.04 \\
\hline División del paisaje-Bosque de encino & 0.04 \\
\hline
\end{tabular}

Valores en negrita indican coeficientes de correlación significativos (P $<0.05)$.

\section{DISCUSIÓN}

\section{Análisis de imágenes de satélite}

En el contexto del análisis del paisaje, la información obtenida por sensores montados en plataformas espaciales constituye una fuente de datos invaluable para describir la condición y patrones espacio-temporales del paisaje a diversas escalas (Horning, Robinson, Sterling, Turner y Spector, 2010). Un factor importante a considerar cuando se clasifica la vegetación y el uso del suelo a partir de imágenes de satélite es la cuantificación del error de clasificación. Para el caso de este estudio, se alcanzaron fiabilidades entre $88 \%$ y $91 \%$, lo que se encuentra cercano a los valores especificados por Anderson, Hardy, Roach y Witmer (1976), que sugieren que la exactitud global de la clasificación debe ser de al menos $90 \%$. En este sentido, las clasificaciones fueron satisfactorias y es posible determinar los cambios en los tipos de vegetación considerados y establecer relaciones entre el paisaje y los incendios forestales en el área de estudio. Sin embargo, los resultados presentados deben interpretarse en el contexto del error derivado de las clasificaciones, ya que pueden tener hasta $12 \%$ de error como resultado de la confusión entre tipos de

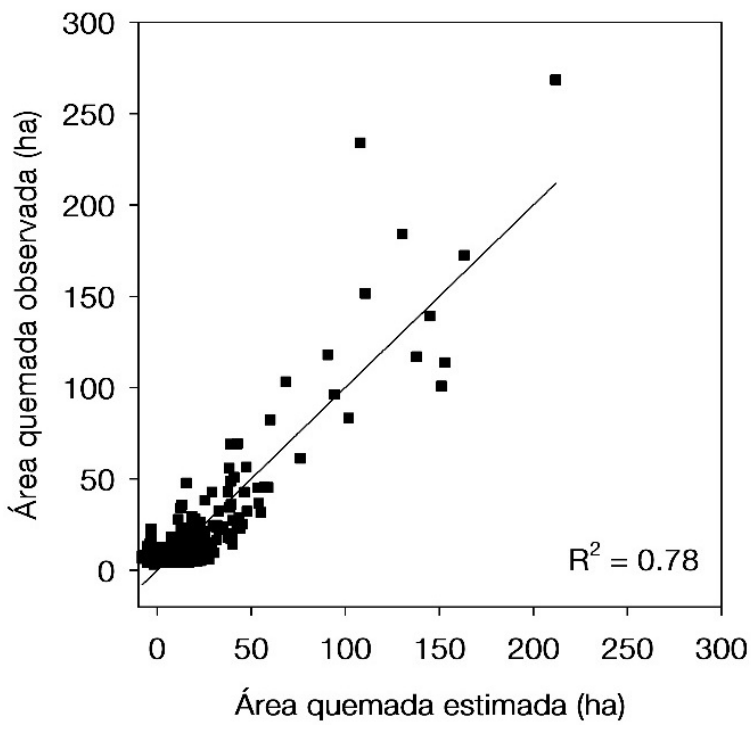

FIGURA 6. Área quemada observada contra área quemada estimada por el modelo de regresión lineal. $\mathrm{n}=383$. 
vegetación. Asimismo, el área total incendiada anualmente, resultante la delimitación de los perímetros de incendios con el programa BAMS está correlacionada con el área total incendiada registrada por la Conafor en el municipio de Madera, lo que demuestra la utilidad de este programa de cómputo para sistematizar la delimitación de áreas quemadas a partir de imágenes Landsat.

\section{Cambios en el paisaje causados por incendios forestales}

El aumento del número de parches y la disminución de su área en los paisajes posincendio sugiere una mayor fragmentación dentro de los polígonos incendiados en el municipio de Madera. Asimismo, el incremento de la división del paisaje fortalece la idea de la fragmentación, al existir un mayor espacio de separación y una mayor discontinuidad entre parches. Estos resultados coinciden con lo descrito por Bizama et al. (2011) y Lloret et al. (2002), quienes encontraron que el paisaje se fragmentó al aumentar el número de parches de menor área después de los incendios. Por otra parte, Chapa, Sosa y de Alba (2008), encontraron que la fragmentación del paisaje compuesto por bosques de pino y de encino aumentó después de los incendios en el estado de Aguascalientes.

En general, el paisaje tiende a ser más heterogéneo (menor número de parches, pero de mayor tamaño) después de la ocurrencia de incendios en comparación con la condición preincendio, tal y como se planteó en la hipótesis de este trabajo. Este resultado sustenta la noción de que los paisajes preincendio son más homogéneos y más susceptibles de quemarse (Lloret et al., 2002) y, cuando ocurren incendios, se vuelven más heterogéneos (PérezCabello et al., 2007; Vega-García y Chuvieco, 2006; Viedma, Moreno y Rieiro, 2006).

La disminución en el área promedio de parches y en el índice del parche más grande puede deberse a que la cantidad de calor liberada durante el incendio tiene una relación directa con las diferencias observadas en la severidad del incendio (Vieira, Fernández, Vega y Keizer, 2015). En virtud de que una severidad alta está relacionada con la destrucción parcial o total de la materia orgánica, la cantidad de vegetación y cobertura superficial del suelo se reduce (Keeley, 2009), lo que ocasiona alteraciones diferentes en el paisaje en función del tipo de vegetación (Clarke, Knox, Bradstock, Muñoz-Robles y Kumar, 2014). La conectividad del combustible forestal es también un factor importante a considerar, ya que el combustible puede tener discontinuidad y dar lugar a parches sin quemar o viceversa (Turner y Romme, 1994).

\section{Relación paisaje preincendio y tamaño de incendios forestales}

En el área de estudio se puede esperar que la homogeneidad del paisaje preincendio favorezca la propagación del fuego, al existir parches grandes de bosque de pino y bosque de pino-encino, que son tipos de vegetación altamente combustibles. El análisis de correlación indica que la homogeneidad del paisaje aumenta la propagación del fuego, lo que resulta en perímetros incendiados de mayor tamaño, lo que coincide con lo encontrado por Lloret et al. (2002). Además de las características del paisaje preincendio, Silva et al. (2011), mencionan que otras propiedades del fuego, como su intensidad, frecuencia y severidad influyen en los cambios del paisaje y es necesario incluirlas en la predicción de la magnitud y tamaño de las áreas incendiadas. La integración de los resultados de este estudio puede usarse para identificar los paisajes altamente vulnerables a incendios, que dadas sus características del paisaje preincendio, pueden influir en la ocurrencia y el tamaño de los incendios. Por ejemplo, en el contexto del manejo del fuego, es posible cuantificar a nivel de rodal o de microcuenca el número y el área de parches de vegetación susceptible $y$, con esto, identificar áreas con mayor probabilidad de ocurrencia de incendios de cierto tamaño. A nivel de paisaje, la ocurrencia de incendios y propagación del fuego es el resultado de interacciones complejas entre las fuentes de ignición, el clima, la topografía y la cubierta del suelo (Mermoz, Kitzberger y Veblen, 2005). De estas variables, la cubierta del suelo, que guarda relación con la estructura de la vegetación y las cargas de combustibles forestales, es la única que puede ser manipulada. De esta manera, la heterogeneidad del paisaje 
puede manejarse para disminuir la propagación del fuego. En el área de estudio, el conocimiento de esta relación puede ayudar a definir medidas de manejo del fuego a nivel de paisaje con la finalidad de hacer que los paisajes sean menos propensos a incendios forestales (Moreira et al., 2011). Lo anterior puede lograrse mediante acciones que interrumpan la continuidad de los combustibles con base en la relación entre las cargas de combustibles forestales y el arreglo espacial de los tipos de vegetación que conforman el paisaje.

\section{CONCLUSIONES}

Los incendios forestales en la región de Madera, Chihuahua originan paisajes más fragmentados y con menor conectividad debido al aumento del número de parches, la división del paisaje y la disminución del área promedio de parches y del parche más grande.

Se obtuvo evidencia de que después de la ocurrencia de incendios los paisajes en la región son más heterogéneos y que el tamaño de los incendios está relacionado con las características espaciales de los paisajes preincendio tales como área promedio y número de parches.

Si bien el efecto del paisaje en la incidencia y tamaño de incendios forestales no es el único factor a considerar, es de gran utilidad para integrarse en modelos más complejos donde se combinen variables ambientales (clima, topografía), socioeconómicas (actividades económicas, densidad de población, infraestructura de caminos) para identificar áreas vulnerables a incendios y que fundamenten el uso ecológico del fuego.

\section{RECONOCIMIENTOS}

Al Consejo Nacional de Ciencia y Tecnología (CONACy'T) por el otorgamiento de la beca (Becario no. 334012) a G. A. A. para la realización de esta investigación.

\section{REFERENCIAS}

Anderson, J. R., Hardy, E. E., Roach, J. T., \& Witmer, R. E. (1976). A land use and land cover classification system for use with remote sensor data. Washington, D.C.: U.S. Geological Survey.
Arenas, S., Haeger, J. F., \& Jordano, D. (2011). Aplicación de técnicas de teledetección y GIS sobre imágenes Quickbird para identificar y mapear individuos de peral silvestre (Pyrus bourgeana) en bosque esclerófilo mediterráneo. Revista de Teledetección, 35, 55-71.

Bajocco, S., \& Ricotta, C. (2008). Evidence of selective burning in Sardinia (Italy): wich land cover classes do wildfires prefer? Landscape Ecology, 23(2), 241-248. doi: 10.1007/s10980-007-9176-5

Bastarrika, A., Alvarado, M., Artano, K., Martinez, M. P., Mesanza, A., Torre, L., Ramo, R., \& Chuvieco, E. (2014). BAMS: A Tool for Supervised Burned Area Mapping Using Landsat Data. Remote Sensing, 6(12), 12360-12380. doi: $10.3390 /$ rs61212360

Bizama, G., Torrejón, F., Aguayo, M., Muñoz, M., Echeverría, C., \& Urrutia, R. (2011). Pérdida y fragmentación del bosque nativo en la cuenca del río Aysén (Patagonia-Chile) durante el siglo XX. Revista de Geografía Norte Grande, 49, 125-138. doi: 10.4067/S0718-34022011000200008

Burgan, R. E., Klaver, R. W., \& Klaver, J. M. (1998). Fuel models and fire potential from satellite and surface observations. International Journal of Wildland Fire, 8(3), 159-170. doi: 10.1071/WF9980159

Clarke, P. J., Knox, K. J. E., Bradstock, R. A., Muñoz-Robles, C., \& Kumar, L. (2014). Vegetation, terrain and fire history shape the impact of extreme weather on fire severity and ecosystem response. Journal of Vegetation Science, 25(4), 10331044. doi: $10.1111 /$ jvs.12166

Conafor. (2016). Reportes de incendios forestales (1999-2014). Recuperado de http://www.conafor.gob.mx/web/temasforestales/incendios/

Chapa, B., Sosa, R., \& de Alba, Á. (2008). Estudio multitemporal de fragmentación de los bosques en la Sierra Fría, Aguascalientes, México. Madera y Bosques, 14(1), 37-51. doi: 10.21829/myb.2008.1411216

Chuvieco, E. (1996). Fundamentos de teledetección espacial. Madrid: Ediciones Rialp.

Di Bella, C. M., Posse, G., Beget, M. E., Fischer, M. A., Mari, N., \& Veron, S. (2008). La teledetección como herramienta para la prevención, seguimiento y evaluación de incendios e inundaciones. Ecosistemas, 17(3), 39-52.

Flores, G., \& Benavides, S. J. (1994). Algunas condiciones que influyen en el riesgo y peligrosidad de los incendios forestales. México: SARH. INIFA. 
Forman, R., \& Godron, M. (1986). Landscape Ecology. New York, USA: Wiley and Sons.

García, E. (1973). Modificaciones al sistema de clasificación climática de Köppen. México, D. F.: UNAM.

Green, B. H., Simmons, E. A., \& Woltjer, I. (1996). Landscape conservation. Some steps towards developing a new conservation dimension $A$ draft report of the IUCN-CESP Landscape Conservation Working Group. Ashford, Kent, UK: Department of Agriculture, Horticulture and Environment.

Haire, S. L., \& McGarical, K. (2010). Effects of landscape patterns of fire severity on regenerating ponderosa pine forests (Pinus ponderosa) in New Mexico and Arizona, USA. Landscape Ecology, 25(7), 1055-1069. doi: 10.1007/s10980010-9480-3

Horning, N., Robinson, J. A., Sterling, E. J., Turner, W., \& Spector, S. (2010). Remote Sensing for Ecology and Conservation. New York, USA: Oxford University Press.

Instituto Nacional de Estadística y Geografía [Inegi] (2003). Síntesis de Información Geográfica del estado de Chihuahua. Aguascalientes, México.

Ireland, K. B., Stan, A. B., \& Fulé, P. Z. (2012). Bottom-up control of a northern Arizona ponderosa pine forest fire regime in a fragmented landscape. Landscape Ecology, 27(7), 983-997. doi: 10.1007/s10980-012-9753-0

Jung, M. (2016). LecoS - A python plugin for automated landscape ecology analysis. Ecological Informatics, 31, 18-21. doi: 10.1016/j.ecoinf.2015.11.006.

Keeley, J. E. (2009). Fire intensity, fire severity and burn severity: a brief review and suggested usage. International Journal of Wildland Fire, 18(1), 116-126. doi: 10.1071/WF07049

Lavorel, S., Flanningan, M. D., Lambin, E. F., \& Scholes, M. C. (2007). Vulnerability of land systems to fire: Interactions among humans, climate, the atmosphere, and ecosystems. Mitigation and Adaptation Strategies for Global Change, 12(1), 33-53. doi: 10.1007/s11027-006-9046-5

Lloret, F., Calvo, E., Pons, X., \& Díaz-Delgado, R. (2002). Wildfires and landscape patterns in the Eastern Iberian Peninsula. Landscape Ecology, 17(8), 745-759. doi: 10.1023/A:1022966930861

Masek, J. G., Goward, S. N., Kennedy, R. E., Cohen, W. B., Moisen, G. G., Schleeweis, K., \& Huang, C. (2013). United States forest disturbance trends observed using Landsat time series. Ecosystems, 16(6), 1087-1104. doi: 10.1007/s10021-013-9669-9
Mermoz, M., Kitzberger, T., \& Veblen, T. T. (2005). Landscape influences on occurrence and spread of wildfires in Patagonian forests and shrublands. Ecology, 86(10), 27052715. doi: $10.1890 / 04-1850$

Moreira, F., Viedma, O., Arianoutsou, M., Curt, T., Koutsias, N., Rigolot, E., Barbati, E., Corona, P., Vaz, P., Xanthopoulos, G., Mouillot, F., \& Bilgili, E. (2011). Landscape-wildfire interactions in southern Europe: Implications for landscape management. Journal of Environmental Management, 92(10), 2389-2401. doi: 10.1016/j.jenvman.2011.06.028

Palacio, J. L., Bocco, G., Velásquez, A., Mas, J. F., Takaki, F., Victoria, A., ..., \& González, F. (2000). La condición actual de los recursos forestales en México: resultados del inventario forestal nacional 2000. Investigaciones Geográficas, 43, 183-203.

Parisien, M. A., Miller, C., Ager, A. A., \& Finney, M. A. (2010). Use of artificial landscapes to isolate controls on burn probability. Landscape Ecology, 25(1), 79-93. doi: 10.1007/s10980-009-9398-9

Pérez-Cabello, F., Ibarra, P., De La Riva, J., Echeverría, M. T., Montorio, R., \& García-Martín, A. (2007). Impacto de los incendios forestales en comunidades vegetales submediterraneas: Evaluación multitemporal de la diversidad del paisaje utilizando imágenes LANDSAT TM. Cuaderno de Investigación Geográfica (33), 101-114.

R Development Core Team. (2015). R: A language and environment for statistical computing. Vienna. http://www.R-project.org: R Foundation for Statistical Computing.

Robichaud, P. R., Beyers, J. L., \& Neary, D. G. (2000). Evaluating the efictiveness of postfire rehabilitation treatments General Technical Report RMRS-GTR-63 (Vol. General Technical Report RMRS-GTR-63, pp. 85). Fort Collins, CO, USA: USDA Forest Service, Rocky Mountain Research Station.

Roy, D., \& Landmann, T. (2005). Characterizing the surface heterogeneity of fire effects using multi-temporal reflective wavelength data. International Journal of Remote Sensing, 26(19), 4197-4218. doi: 10.1080/01431160500112783

Silva, J. S., Vaz, P., Moreira, F., Catry, F., \& Rego, F. (2011). Wildfires as a major driver of landscape dynamics in three fire-prone areas of Portugal. Landscape and Urban Planning, 101(4), 349-358. doi: 10.1016/j.landurbplan.2011.03.001

Turner, M. G., \& Romme, W. H. (1994). Landscape dynamics in crown fire ecosystems. Landscape Ecology, 9(1), 59-77. doi: 10.1007/BF00135079 
United States Department of the Interior (s/f). USGS Global Visualization Viewer. Recuperado de https://glovis.usgs.gov/

Vázquez de la Cueva, A., \& Rodríguez Martín, J. A. (2008). Dinámica de paisajes forestales en relación a la incidencia del fuego en España peninsular: 1987-2000. Investigación Agraria. Sistemas y Recursos Forestales, 17(2), 143-154.

Vega-García, C., \& Chuvieco, E. (2006). Applying local measures of spatial heterogeneity to Landsat-TM images for predicting wildfire occurrence in Mediterranean landscapes. Landscape Ecology, 21(4), 595-605. doi: 10.1007/s10980-005-4119-5

Viedma, O., Moreno, J. M., \& Rieiro, I. (2006). Interactions between land use/land cover change, forest fires and landscape structure in Sierra de Gredos (Central Spain). Environmental Conservation, 33(3), 212-222. doi: 10.1017/S0376892906003122

Vieira, D. C. S., Fernández, C., Vega, J. A., \& Keizer, J. J. (2015). Does soil burn severity affect the post-fire runoff and interrill erosion response? A review based on meta-analysis of field rainfall simulation data. Journal of Hydrology, 523, 452-464. doi: 10.1016/j.jhydrol.2015.01.071

Manuscrito recibido el 8 de enero de 2018

Aceptado el 19 de abril de 2018

Publicado el 9 de noviembre de 2018

Este documento se debe citar como:

Alva-Álvarez, G. I., Reyes-Hernández, H., Palacio-Aponte, A. G., Núñez-López, D., \& Muñoz-Robles, C. (2018). Cambios en el paisaje ocasionados por incendios forestales en la región de Madera, Chihuahua. Madera y Bosques, 24(3), e2431697. doi: 10.21829/myb.2018.2431697

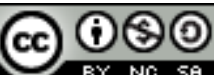

Madera y Bosques por Instituto de Ecología, A.C. se distribuye bajo una Licencia Creative Commons Atribución-NoComercialCompartirlgual 4.0 Internacional. 